\title{
Diagnóstico Quali-Quantitativo dos Resíduos Sólidos Gerados no Campus V da Universidade do Estado do Pará
}

\author{
Quali-quantitative Diagnosis of Solid Waste Generated at Campus V of the State University of Pará \\ Diagnóstico Cualitativo y Cuantitativo de Residuos Sólidos Generados en el Campus V de la \\ Universidad Estatal de Pará
}

Recebido: 25/08/2021 | Revisado: 30/08/2021 | Aceito: 08/09/2021 | Publicado: 11/09/2021

\author{
Heloiza Santos Borges \\ ORCID: https://orcid.org/0000-0002-6846-419X \\ Universidade do Estado do Pará, Brasil \\ E-mail:santosheloiza@gmail.com \\ Maria Eduarda Assunção Ogorodnik \\ ORCID: https://orcid.org/0000-0001-8092-4809 \\ Universidade do Estado do Pará, Brasil \\ E-mail:eduardaogorodnik@gmail.com \\ Leonardo Silva do Nascimento \\ ORCID: https://orcid.org/0000-0001-5336-5757 \\ Universidade do Estado do Pará, Brasil \\ E-mail: leonardolv21@gmail.com \\ Eder Silva de Oliveira \\ ORCID: https://orcid.org/0000-0002-2560-2214 \\ Universidade do Estado do Pará, Brasil \\ E-mail: ederso@uepa.br \\ Denise Cristina Torres Costa \\ ORCID: https://orcid.org/0000-0002-3478-1656 \\ Universidade do Estado do Pará, Brasil \\ E-mail denise.costa@uepa.br
}

\begin{abstract}
Resumo
A geração de resíduos sólidos está relacionada diretamente com as atividades humanas, seu adequado gerenciamento é fundamental para a preservação do meio ambiente. Para a elaboração de planos e projetos relacionados ao gerenciamento dos resíduos sólidos é necessário, primordialmente, a realização do diagnóstico quali-quantitativo de resíduos no local onde vai ser implementado o plano. Desse modo, o presente trabalho teve como objetivo realizar o diagnóstico quali-quantitativo dos resíduos gerados no Campus V - Centro de Ciências Naturais e Tecnologia (CCNT) da Universidade do Estado do Pará e propor estratégias para o melhor gerenciamento destes resíduos. A análise qualiquantitativa ocorreu durante o período de uma semana letiva, o diagnóstico quantitativo foi efetuado através do método de quarteamento descrito na NBR 10007:2004 e a classificação dos resíduos for realizada de acordo com a NBR 10.004:2004. Os resultados demonstram que o Campus V da UEPA produz, em média, 86,26 kg de resíduos sólidos semanalmente, com uma geração per capta de $0,098 \mathrm{~kg} / \mathrm{hab} / \mathrm{dia}$. O resíduo que se apresentou em maior concentração, nas amostras realizadas, foi o orgânico (40\%), seguido do papel/papelão $(35,6 \%)$ e do plástico $(16,9 \%)$. Dentre as alternativas para gerenciar os resíduos estão a elaboração e implementação de um Plano de Resíduos Sólidos (PGRS), a promoção da educação ambiental, a implantação da compostagem na universidade e a realização de parcerias com cooperativas e associações de reciclagem.
\end{abstract}

Palavras-chave: Resíduos sólidos; Universidade; Gerenciamento de resíduos sólido.

\begin{abstract}
The generation of solid waste is directly related to human activities, its adequate management is fundamental for the preservation of the environment. For the preparation of plans and projects related to the management of solid waste, it is necessary, primarily, to carry out a quali-quantitative diagnosis of waste in the place where the plan will be implemented. Thus, the present work aimed to carry out a qualitative and quantitative diagnosis of the waste generated at Campus V - Center for Natural Sciences and Technology (CCNT) of the University of the State of Pará and to propose strategies for the better management of this waste. The quali-quantitative analysis took place during the period of one academic week, the quantitative diagnosis was made through the method of quartering described in NBR 10007: 2004 and the classification of residues is carried out according to NBR 10.004: 2004. The results demonstrate that UEPA Campus V produces, on average, $86.26 \mathrm{~kg}$ of solid waste weekly, with a per capita generation of $0.098 \mathrm{~kg} / \mathrm{inhab} /$ day. The residue that presented the highest concentration in the samples carried out was organic (40\%), followed by paper /
\end{abstract}


cardboard (35.6\%) and plastic (16.9\%). Among the alternatives for managing waste are the preparation and implementation of a Solid Waste Plan (PGRS), the promotion of environmental education, the implementation of composting at the university and the establishment of partnerships with cooperatives and recycling associations.

Keywords: Solid waste; University; Solid waste management.

\section{Resumen}

La generación de residuos sólidos está directamente relacionada con las actividades humanas, su adecuado manejo es fundamental para la preservación del medio ambiente. Para la elaboración de planes y proyectos relacionados con el manejo de residuos sólidos, es necesario, principalmente, realizar un diagnóstico cuali-cuantitativo de residuos en el lugar donde se implementará el plan. Así, el presente estudio tuvo como objetivo realizar un diagnóstico cualitativo y cuantitativo de los residuos generados en el Campus V - Centro de Ciencias Naturales y Tecnología (CCNT) de la Universidad del Estado de Pará y proponer estrategias para la mejor gestión de estos residuos. El análisis cualicuantitativo se realizó durante el período de una semana académica, el diagnóstico cuantitativo se realizó mediante el método de despiece descrito en NBR 10007: 2004 y la clasificación de residuos se realiza según NBR 10.004: 2004. Los resultados muestran que el Campus V de la UEPA produce, en promedio, 86,26 kg de residuos sólidos semanales, con una generación per cápita de $0,098 \mathrm{~kg} /$ hab / día. El residuo que presentó mayor concentración en las muestras realizadas fue el orgánico (40\%), seguido del papel / cartón (35,6\%) y el plástico (16,9\%). Entre las alternativas para el manejo de residuos se encuentran la elaboración e implementación de un Plan de Residuos Sólidos (PGRS), la promoción de la educación ambiental, la implementación del compostaje en la universidad y el establecimiento de alianzas con cooperativas y asociaciones de reciclaje.

Palabras clave: Residuos sólidos; Universidad; Manejo de residuos sólidos.

\section{Introdução}

O mundo sempre esteve em constante mudança, desde épocas remotas, anteriores à humanidade, até as grandes revoluções na indústria e no modo de exploração dos recursos naturais. Sendo assim, em se tratando de história do homem, as sociedades mais antigas encontravam formas de criar suas ferramentas para o seu sustento. Ao longo das eras, a maneira que o ser humano lidava com os recursos naturais veio sofrendo mudanças, e junto delas, os impactos ao meio socioambiental (Albuquerque, 2007). O que antes era visto como um bem essencial à vida, hoje pode ser considerado apenas mais um bem comum. Segundo Baptista (2010, p. 9) "Há milhares de anos atrás o ser humano utilizava os recursos naturais exclusivamente como meio de subsistência, sem outras intenções. Ele ainda não tinha o conhecimento necessário para transformar em bens aquilo que possuía em mãos".

Entretanto, ao descobrir os novos valores que os recursos naturais poderiam ter em suas mãos, o ser humano deixou de ser um mero elemento da paisagem natural em uma interação amigável de subsistência, e passou a estabelecer uma relação de posse e domínio com os recursos naturais (Rodrigues et al, 2019). Essa nova relação se intensificou com o desenvolvimento industrial, onde grandes quantidades de produtos passaram a ser produzidos com maior velocidade, e, junto da evolução da logística, chegaram aos seres humanos com maior rapidez (Andrade, 2017). Assim, tem-se que, atualmente, a ideia de consumo já não se limita apenas ao que serve para a subsistência, mas também para o conforto pessoal e social. Em relação à natureza, são muitos os problemas ecológicos resultantes das formas de consumo de energia, de matéria-prima e, principalmente, dos rejeitos dos produtos eliminados no ambiente (Pereira \& Horn, 2009).

As vicissitudes relacionadas à geração de resíduos ocorrem devido ao meio ambiente ter que assimilar uma grande quantidade de bens descartados enquanto sofre a exploração de seus recursos. Um agravante a esta situação é o fato de que a população mundial aumenta cada vez mais e os bens que antes eram constituídos basicamente de matéria orgânica foram substituídos por bens constituídos de plástico e por outros materiais com maior tempo de degradação, como vidros, metais e polímeros (Andreoli, 2014). Desse modo, pode-se dizer que o desenvolvimento industrial juntamente com o crescimento populacional e a procura cada vez maior por bens de consumo descartáveis têm provocado grandes problemas ambientais (Cajaiba \& Silva, 2016). Com isso, nota-se que na gestão de resíduos, a colaboração da sociedade é algo essencial, porque ela é responsável pela geração de uma quantidade significativa deste (Coutinho et al., 2020). 
O gerenciamento inadequado dos resíduos sólidos urbanos gera impactos tanto ambientais quanto na saúde da população (Gouveia, 2012). Esses impactos podem ser imediatos e/ou a longo prazo, dentre os imediatos tem-se o incômodo gerado pelo odor característico dos resíduos em decomposição e a obstrução de sistemas de microdrenagem causando enchentes e alagamentos. Concernente aos impactos de longo prazo, têm-se que a disposição inadequada dos Resíduos Sólidos Urbanos (RSU) reflete diretamente na saúde pública, uma vez que, na ausência de uma gestão adequada dos mesmos, existe a possibilidade de ocorrer a poluição ambiental e a proliferação de vetores (Mota, 2009).

O correto manejo dos resíduos sólidos gerados se apresenta como um grande desafio no Brasil, de acordo com a Associação Brasileira de Empresas de Limpeza Pública e Resíduos Especiais (ABRELPE) em 2019 foi gerado no Brasil 79 milhões de toneladas de Resíduos Sólidos Urbanos (RSU), sendo que desse valor 92\% (72,7 milhões de toneladas) foi coletado, desse percentual apenas 43,3 milhões de toneladas (59,5\%) receberam destinação adequada em aterros sanitários, e as 29,5 milhões de toneladas restantes $(40,5 \%)$ foram despejadas em locais inadequados. A região Norte, maior região do Brasil em extensão territorial, gerou em 2019 5,8 milhões de toneladas de RSU, das quais apenas 81,31\% foram coletadas. Do total coletado nos 450 municípios da região, mais de 1,6 milhões de toneladas foram parar em lixões, não recebendo a destinação ambientalmente adequada (ABRELPE, 2020).

Para auxiliar o país no enfrentamento dos principais problemas ambientais, sociais e econômicos decorrentes do manejo inadequado dos resíduos sólidos foi criada a lei n ${ }^{\circ}$ 2.305/10 que instituiu a Política Nacional dos Resíduos Sólidos (PNRS). A PNRS prevê a prevenção e a redução na geração de resíduos, tendo como proposta a prática de hábitos de consumo sustentável e um conjunto de instrumentos para propiciar o aumento da reciclagem e da reutilização dos resíduos sólidos e a destinação ambientalmente adequada dos rejeitos. São instrumentos da Política Nacional de Resíduos Sólidos, entre outros: os planos de resíduos sólidos, a coleta seletiva, o incentivo à criação e ao desenvolvimento de cooperativas ou de outras formas de associação de catadores de materiais reutilizáveis e recicláveis, a pesquisa científica e tecnológica e a educação ambiental (Brasil, 2010).

A educação ambiental é um instrumento importante para redução da produção de resíduos, além de conscientizar e definir o papel do que é ecologicamente correto para o meio ambiente, ela dissemina informações essenciais para práticas futuras como reduzir o consumo, reutilizar e reciclar os materiais (Cotica \& Carniatto, 2020). De acordo com o artigo $1^{\circ}$ e $2^{\circ}$ da Política Nacional de Educação Ambiental (Lei n. 9.795, 1999),

$1^{\circ}$ Entendem-se por educação ambiental os processos por meio dos quais o indivíduo e a coletividade constroem valores sociais, conhecimentos, habilidades, atitudes e competências voltadas para a conservação do meio ambiente, bem de uso comum do povo, essencial à sadia qualidade de vida e sua sustentabilidade.

$2^{\circ}$ A educação ambiental é um componente essencial e permanente da educação nacional, devendo estar presente, de forma articulada, em todos os níveis e modalidades do processo educativo, em caráter formal e não-formal (Lei n. 9.795, 1999).

Assim, dada a relevância da educação ambiental, tem-se que as instituições de ensino devem introduzi-la como componente essencial para formação do aluno. Dentre as instituições de ensino, a Universidade se apresenta como um ambiente destinado à formação de indivíduos não somente voltados às áreas técnicas, mas também a formar indivíduos capazes de entender suas responsabilidades diante dos bens de uso comum e da natureza. Dessa forma, de acordo com Jacobi (2003),

A educação ambiental, nas suas diversas possibilidades, abre um estimulante espaço para repensar práticas sociais e o papel dos professores como mediadores e transmissores de um conhecimento necessário para que os alunos adquiram uma base adequada de compreensão essencial do meio ambiente global e local, da interdependência dos problemas e soluções e da importância da responsabilidade de cada um para construir uma sociedade planetária mais eqüitativa e ambientalmente sustentável (Jacobi, 2003, p. 206).

Mas, para que isso ocorra, as universidades devem buscar formas de institucionalizar os valores ambientais em seu cotidiano. Isso pode ocorrer através da adaptação de estruturas que facilitem as práticas ambientais, entre elas pode-se citar: 
implantação de lixeiras para coleta seletiva, instalação de composteiras e quadros educativos que estimulem a redução do uso de materiais mais descartados (plásticos, papéis, metais etc.).

Obter uma abordagem maior desse problema nas universidades é um desafio, pois nem todos os cursos possuem essa abordagem curricular em sua grade. "A universidade deve inserir a discussão das questões ambientais e de educação ambiental nos currículos dos cursos de graduação e pós-graduação e almejar a formação de cidadãos comprometidos com o desenvolvimento de sociedades sustentáveis" (Silva, Marcomin, Mazzuco \& Becker, 2011). Para que isso se concretize, faz-se necessário o comprometimento do corpo docente na inclusão desse tema em suas disciplinas, e posteriormente em mais cursos. Um estudo realizado na Universidade Regional de Blumenau com professores de licenciatura demonstrou que apenas 54\% dos professores já trabalharam com educação ambiental, enquanto 29\% responderam que não (Verdi \& Pereira, 2006). Sendo, então, possível verificar que mesmo em áreas de formação de docentes, licenciaturas, ainda há uma abordagem deficiente sobre educação ambiental nas universidades.

Uma forma de implementar a educação ambiental de maneira articulada e de modo a envolver toda a comunidade acadêmica é através da implementação de um Plano de Gerenciamento de Resíduos Sólidos (PGRS) nas universidades. A elaboração de um plano de gestão de resíduos para unidades de ensino permite prevenir e minimizar problemas relacionados à poluição ambiental, além de promover educação ambiental dentro da unidade universitária, visando à diminuição da produção de resíduos e evitando desperdícios (Mesquita, Fiuza \& Sartori, 2011). O PGRS é um instrumento da PNRS e através dele os resíduos receberão o manejo e destinação final ambientalmente adequados, além disso serão desenvolvidas práticas que estimulem a redução da produção de resíduos e o aumento da reutilização e reciclagem.

O primeiro passo para a elaboração do PGRS é a caracterização quali-quantitativa dos resíduos gerados, que pode ser feita através da gravimetria. A composição gravimétrica pode variar em função de vários fatores, tais como, condições sociais, atividades econômicas, tipos de serviços, clima, geografia, cultura, e vários outros (Massukado, 2004). Considerando que a realização da caracterização quali-quantitativa dos resíduos é fundamental para auxiliar na elaboração de planos e projetos relacionados à gestão de resíduos, o presente trabalho teve como objetivo realizar o diagnóstico quali-quantitativo dos resíduos sólidos produzidos no Centro de Ciências Naturais e Tecnologias (CCNT) - Campus V da Universidade do Estado do Pará.

\section{Metodologia}

\subsection{Tipo de Pesquisa}

Trata-se de um estudo de caso, método que permite a aquisição de subsídios para tomadas de decisão em relação ao fenômeno em estudo (Pereira et al, 2018). A pesquisa é de caráter exploratório, isto é, uma investigação empírica cuja finalidade é desenvolver hipóteses e aumentar o conhecimento acerca de um ambiente, fato ou fenômeno para a realização de uma pesquisa mais precisa (Marconi \& Lakatos, 2003). O estudo possui abordagem quantitativa e qualitativa e, para a obtenção dos resultados, foi efetuada uma pesquisa de campo que, segundo Marconi \& Lakatos (2003) "consiste na observação de fatos e fenômenos tal como ocorrem espontaneamente, na coleta de dados a eles referentes e no registro de variáveis que se presume relevantes, para analisá-los" (Marconi \& Lakatos, 2003, p. 186).

\section{2 Área de Estudo}

A pesquisa foi realizada no Centro de Ciências Naturais e Tecnologias (CCNT) - Campus V da Universidade do Estado do Pará (Figura 1), localizado na Travessa Dr. Enéas Pinheiro, no 2626 - Marco, Região Metropolitana de Belém - PA, CEP 66095015, de Coordenadas Geográficas 48²7’7,723”W e 1²6’5,577’S. O estudo possui caráter bibliográfico e exploratório de campo, pois foram realizadas pesquisas sobre técnicas de amostragem de resíduos a fim de utilizá-las no processo de caracterização dos resíduos gerados na universidade. 
Figura 1: Localização do Centro de Ciências Naturais e Tecnologias (CCNT).

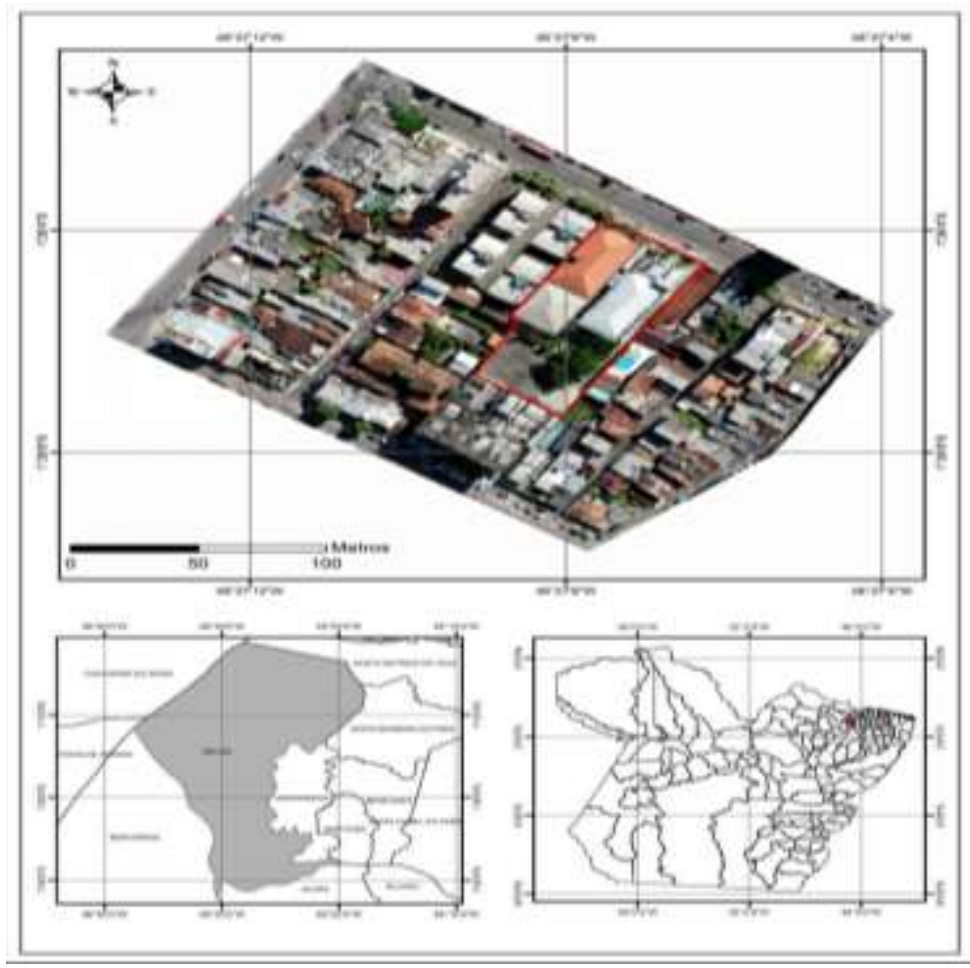

Fonte: Autores.

Os dados referentes ao Campus V da UEPA foram obtidos junto à coordenação da instituição de ensino, desse modo, tem-se que o campus compreende cerca de 880 pessoas entre alunos, servidores e funcionários. Nele são ofertados sete cursos de graduação (Tecnologia de Alimentos, Engenharia de Produção, Design, Engenharia Sanitária e Ambiental, Engenharia Florestal, Tecnologia em Comércio Exterior e Relações Internacionais) e um Programa de Pós-Graduação em Ciências Ambientais em nível de Mestrado Acadêmico e Doutorado. No campus V, também estão presentes seis secretarias de departamento de cursos, oito setores de coordenação dos cursos, sete centros acadêmicos, uma biblioteca e outros trinta e dois setores ativos, entre eles a central de estágio, coordenação administrativa, protocolo, cantina, dentre outros.

\subsection{Coleta e Acondicionamento}

Para a coleta dos resíduos utilizou-se as lixeiras disponíveis na própria universidade, que possui um sistema de coleta seletiva. Estas lixeiras estão distribuídas nos dois prédios principais e conseguem atender a todo o público do campus. A coleta dos resíduos foi realizada pelos funcionários da instituição e ocorreu diariamente. Os resíduos eram retirados dos recipientes menores e acondicionados em um recipiente maior, onde havia apenas a distinção entre resíduos sanitários e comuns.

\subsection{Caracterização Gravimétrica}

A gravimetria dos resíduos, também conhecida como inventário, foi dividida em duas etapas, a quantitativa e a qualitativa. Ela foi realizada no estacionamento do campus, por ser um local acessível e arejado, facilitando assim, o manuseio dos Resíduos Sólidos (RS). Os materiais utilizados para o processo gravimétrico foram acondicionados no Laboratório de Engenharia Ambiental (LEA) e seguem descritos no Quadro 1. 
Quadro 1: Material utilizado na gravimetria.

\begin{tabular}{|c|c|}
\hline Material & Função \\
\hline Balança & Pesagem do RS \\
\hline Barbante & Demarcação dos RS \\
\hline Enxada & Manipulação dos RS \\
\hline Baldes & Para a separação dos RS \\
\hline Pá & Manipulação dos RS \\
\hline Lona plástica & Despejo dos RS \\
\hline Aventais & Proteção no manejo dos RS \\
\hline Álcool em gel & Higienização das mãos \\
\hline Galochas & Proteção no manejo dos RS \\
\hline Luvas & Proteção no manejo dos RS \\
\hline Câmera fotográfica & Coleta dos dados \\
\hline Papel, caneta e calculadora & Coleta dos dados \\
\hline
\end{tabular}

Fonte: Autores.

A caracterização quantitativa foi realizada através da pesagem dos resíduos gerados, por cinco dias úteis consecutivos, calculando-se a média diária, a estimativa média mensal e a composição gravimétrica (Monteiro, 2001). Ao escolher a quantidade de dias para a realização da análise, foram considerados os dias letivos ao longo de uma semana, dessa forma os resíduos foram pesados diariamente durante cinco dias. Essa metodologia permite estimar a produção mensal de resíduos produzidos no campus e, a partir desses dados, estimou-se um quantitativo anual sobre os RS.

Para a pesagem dos resíduos foi utilizada uma balança Welmy modelo BCW 15, com capacidade mínima de 100g e máxima de $15 \mathrm{~kg}$. Durante esta etapa os resíduos foram agrupados em rejeitos e comuns. Os rejeitos representam os resíduos sanitários, que correspondem aos resíduos coletados dos banheiros da instituição que não podem ser reciclados ou reaproveitados e, portanto, devem ser separados dos demais RS. Os resíduos comuns englobam os resíduos recicláveis ou que podem ser reutilizados (papel, metal, vidro, orgânico e plástico).

Na composição gravimétrica foi utilizado o método do quarteamento descrito na ABNT NBR 10007:2004, que tem como objetivo a obtenção de uma amostra representativa, isto é, a coleta de uma parcela do resíduo a ser estudada que, quando analisada, apresenta as mesmas características e propriedades de sua massa total. O quarteamento foi realizado a partir do processo de divisão em quatro partes iguais de uma amostra pré-homogenizada, sendo tomadas duas partes opostas entre si para construir uma nova amostra e descartadas as partes restantes. As partes não descartadas foram misturadas totalmente e o processo de quarteamento é repetido até que se obtenha o volume desejado (ABNT, 2004). 
Após a caracterização quantitativa, os resíduos foram separados em cinco categorias (papel, metal, vidro, orgânico e plástico) para a realização da caracterização qualitativa dos resíduos com base na NBR 10.004:2004 que classifica os resíduos sólidos quanto aos seus riscos potenciais ao meio ambiente e à saúde pública, para que possam ser gerenciados adequadamente. De acordo com essa norma, os resíduos são classificados em duas classes: Classe I - perigosos e classe II - não perigosos, a classe II é subdividida em: classe IIA - não inertes e classe IIB- inertes.

Os resíduos da Classe I, são representados por aqueles que, em função de suas propriedades físicas, químicas ou infectocontagiosas, podem apresentar risco à saúde pública (provocando mortalidade, incidência de doenças ou acentuando seus índices) e riscos ao meio ambiente (quando o resíduo for gerenciado de forma inadequada). Os resíduos da Classe II A são aqueles que não se enquadram nas classificações de resíduos classe I - Perigosos ou de resíduos classe II B - Inertes, estes resíduos podem ter propriedades como biodegradabilidade, combustibilidade ou solubilidade em água. Os resíduos da classe IIB são resíduos que ao serem submetidos ao teste de solubilidade, não possuem nenhum de seus constituintes solubilizados a concentrações superiores aos padrões de potabilidade da água, excetuando-se aspecto, cor, turbidez, dureza e sabor (ABNT, 2004).

Para estimar a produção per capita de resíduos gerados em cada campus realizou-se a divisão da quantidade total de resíduos gerados por semana pela quantidade de pessoas que circulam em cada Campus (equação 1).

Equação 1: Cálculo da geração per capta de resíduos gerados.

$$
\mathrm{GP}=\mathrm{Qr} / \mathrm{P}
$$

GP = geração per capita de resíduos sólidos, Kg.semana- ${ }^{-1}$.pessoa-1 ${ }^{1}$

$\mathrm{Qr}=$ quantidade de resíduos sólidos, produzida por semana, $\mathrm{Kg} / \mathrm{semana}$

$\mathrm{P}=$ quantidade de pessoas que circula no campus

\section{Relato de Caso}

Através do levantamento quantitativo dos RS gerados, tem-se que o CCNT produz semanalmente 86,26 kg de resíduos, dos quais 16,16 kg correspondem aos rejeitos (resíduos sanitários) e 70,1 Kg correspondem ao resíduo comum. Desse modo, estima-se que mensalmente sejam produzidos 345,04 Kg de resíduos advindos das atividades realizadas na instituição. Projetando esses dados para obter uma média anual, estima-se que a universidade produza mais de $4.000 \mathrm{~kg}$ de RS. Vale ressaltar que, essas estimativas estão sujeitas a alterações que dependem do cronograma acadêmico de atividades da instituição.

Em relação à composição gravimétrica, o resíduo que se apresentou em maior quantidade foi o orgânico (40\%) - oriundo da cantina presente no Campus - seguido de papel/papelão (35,6\%) - proveniente, principalmente, das atividades dos setores administrativos e das atividades realizadas em sala de aula - e do plástico (16,9\%) - composto por utensílios utilizados para consumo de alimentos e bebidas como pratos, copos e talheres descartáveis. Os RS que se apresentaram em menor quantidade foram os resíduos de vidro (garrafas e copos quebrados) e de metal (latas de refrigerante e suco), ambos com 1,3\% de representatividade, a categoria outros ocupou $4,9 \%$ de todo o resíduo quantificado. 
Figura 2: Composição Gravimétrica dos resíduos gerados no CCNT-UEPA.

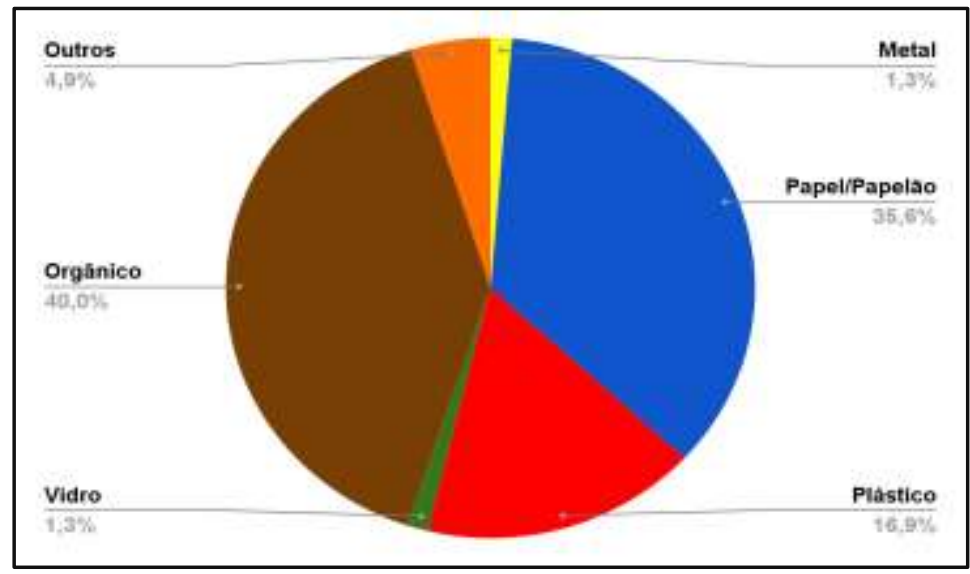

Fonte: Autores.

A partir da gravimetria, tornou-se possível estimar a quantidade de cada tipo de resíduo produzido mensalmente (Figura 3). O resíduo orgânico e o resíduo de papel/papelão foram os que apresentaram maior produção mensal com $112,16 \mathrm{~kg}$ e 99,82 $\mathrm{kg}$ respectivamente. O rejeito (resíduos sanitários) foi o que teve a terceira maior produção mensal $(64,64 \mathrm{~kg})$ na instituição e o resíduo plástico $(47,39 \mathrm{~kg})$ foi o quarto com maior representatividade. Os tipos de resíduos menos produzidos na instituição são o vidro e o metal (ambos com produção mensal de 3,64 kg) e aqueles que são classificados como outros (borracha, caneta, folhagem, madeira e terras oriundas das atividades de varrição do campus), que, devido a sua pouca representatividade individual, foram agrupados em uma categoria somente.

Figura 3: Geração mensal de cada tipo de resíduo.

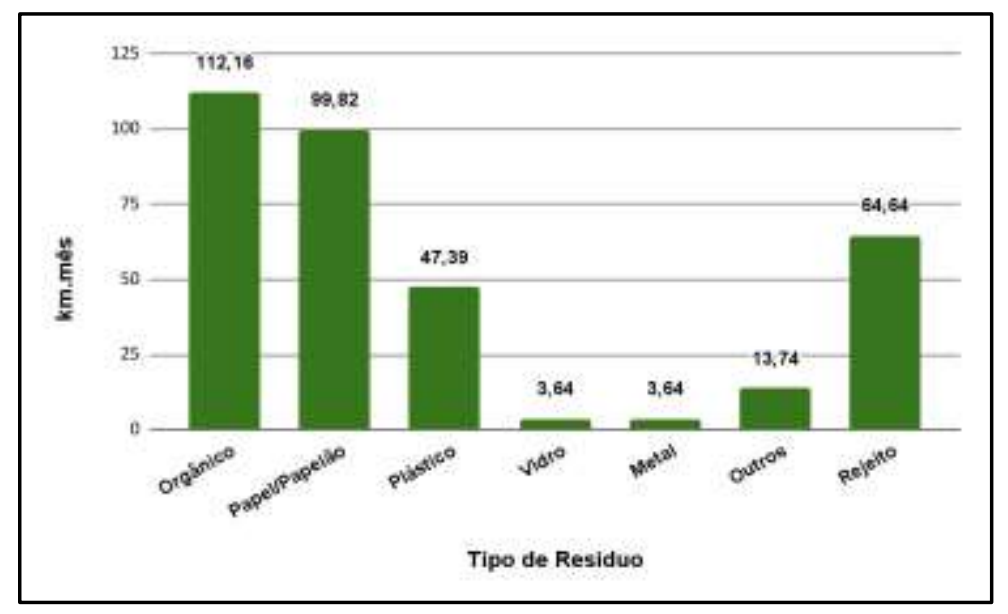

Fonte: Autores.

\section{Discussão}

Ao comparar a quantidade semanal de resíduos produzida no campus V da UEPA com a produzida em Campus de outras universidades (Quadro 2), observa-se que dois fatores são importantes em relação à quantidade de resíduos produzidos nas universidades: a área de ocupação e o número de pessoas que frequentam o campus. O campus Medianeira da UTFPR e o campus I da UEPB possuem áreas maiores com vários blocos contendo salas de aula, laboratórios, lanchonetes e restaurante universitário, bem como maior quantidade de alunos, professores e servidores circulando na instituição, dessa forma, nelas há 
maior geração de resíduos sólidos semanalmente. O campus V da UEPA e o campus IV da UFPB possuem áreas menores comparadas aos demais campus estudados, além disso a quantidade de pessoas que circulam no campus também é menor, reduzindo assim a quantidade de resíduos gerada.

Quadro 2: Comparação da produção semanal de resíduos com outros Campus.

\begin{tabular}{|c|c|c|c|c|}
\hline Universidade & $\begin{array}{c}\text { Área } \\
\text { Construída }\end{array}$ & Kg.semana & $\begin{array}{c}\mathbf{N}^{\mathbf{0}} \text { de pessoas que } \\
\text { frequentam o } \\
\text { campus diariamente }\end{array}$ & kg.sem $^{\mathbf{- 1}}$.pessoa-1 \\
\hline Campus V - UEPA & $5.614,26 \mathrm{~m}^{\mathbf{2}}$ & 86,260 & 880 & 0,098022727 \\
\hline $\begin{array}{c}\text { Campus IV - UFPB } \\
\text { (Lima, 2019) }\end{array}$ & $4.0893 \mathrm{~m}^{\mathbf{2}}$ & 256,785 & 1.348 & 0,190493323 \\
\hline $\begin{array}{c}\text { Campus Medianeira - UTFPR } \\
\text { (Madeira \& Martinelli, 2014) }\end{array}$ & $36.019,70 \mathrm{~m}^{\mathbf{2}}$ & 228,623 & 2.382 & 0,095979429 \\
\hline $\begin{array}{c}\text { Campus I - UEPB } \\
\text { (Costa et al., 2004) }\end{array}$ & $77.908,72$ & 573,160 & 3.327 & 0,172275323 \\
\hline
\end{tabular}

Fonte: Autores.

Ao analisar a produção per capita de resíduos gerados em cada campus, foi observado que o Campus IV da UFPB e o Campus I da UEPB produzem mais resíduos em relação aos outros campus, independente da quantidade de pessoas, o que revela que há outros fatores que interferem na quantidade de resíduos gerados por cada indivíduo, como o nível de educação ambiental, por exemplo. Quanto aos tipos de resíduos gerados, os principais produzidos no CCNT foram: orgânico; papel/papelão; plástico; vidro; metal; outros e rejeito. Esses RS foram classificados de acordo com a ABNT NBR 10004/2004 e tiveram o seu percentual na amostra calculado através da composição gravimétrica (Figura 2).

Ao analisar a quantidade de resíduos gerados mensalmente, tem-se que o resíduo orgânico é o tipo mais produzido pela instituição (112,16 kg), isso ocorre devido ao grande desperdício de alimentos, sendo assim necessária a utilização de estratégias para sensibilização dos alunos e funcionários incentivando o melhor aproveitamento dos alimentos e a redução do desperdício. O resíduo de papel/papelão é o segundo tipo mais produzido pelo Campus V da UEPA $(99,82 \mathrm{~kg})$. Para reduzir essa quantidade é fundamental que os alunos e funcionários entendam a importância da reutilização do papel para preservação do meio ambiente, e essa compreensão se dá através da educação ambiental, que pode ser desenvolvida através de palestras, eventos, e discussões a respeito do tema. O resíduo plástico é o terceiro com maior representatividade mensal (47,39 kg), a redução da geração desse tipo de resíduo pode ocorrer através de campanhas visando a substituição do uso de descartáveis pelo uso de utensílios reutilizáveis.

O resíduo orgânico, que geralmente tem sua destinação final no aterro sanitário, pode se transformar em adubos e fertilizantes através do processo de compostagem. Um estudo realizado na Universidade Tecnológica Federal do Paraná em Campo Mourão (UTFP - Campus Campo Mourão) demonstrou sucesso no uso da composteira para a destinação dos resíduos orgânicos do restaurante universitário, além de folhagens provenientes da manutenção paisagística do campus. No projeto que deu origem a pesquisa, o adubo gerado pela composteira foi utilizado em atividades de educação ambiental em escolas da região, fomentando a sensibilização de alunos da educação básica (Rodrigues, Mattos, Antunes, Souza \& Gonçalves, 2019).

Sendo assim, segundo Carvalho et al. (2012) a compostagem é um instrumento prático e barato que promove a educação ambiental alertando para os riscos da destinação e do manejo inadequado dos resíduos orgânicos. Além disso, a compostagem é uma alternativa financeiramente interessante, pois todo o resíduo que antes não trazia retorno algum, pode servir de insumo para projetos paisagísticos e de manutenção da universidade economizando custos. Um diagnóstico feito na Universidade Federal de Santa Maria, no Campus de Palmeira das Missões, mostrou que a implementação da composteira pode gerar $1140 \mathrm{~kg}$ de adubo a cada três meses, economizando cerca de $\mathrm{R} \$$ 8.493,00 em compras de sacos de $20 \mathrm{~kg}$ de adubo (Stamm et al. 2019). 
Os demais resíduos produzidos na instituição (papel, plástico e metal), devem ser separados e destinados às cooperativas de coleta seletiva. Um dos instrumentos da PNRS é a criação e o desenvolvimento de cooperativas ou de outras formas de associação de catadores de materiais reutilizáveis e recicláveis, desse modo, destinar os resíduos recicláveis às cooperativas é uma forma de contribuir para o gerenciamento correto dos resíduos produzidos no Campus.

Entretanto, para que a coleta seletiva seja implementada de modo adequado, é imprescindível que ocorra a promoção da educação ambiental, que também é um dos instrumentos da PNRS, através dela os alunos e funcionários da instituição serão instruídos a respeito dos impactos negativos decorrentes do descarte inadequado de resíduos e serão orientados a respeito de práticas visando a não geração, a redução, a reutilização e a reciclagem de resíduos sólidos.

\section{Conclusão}

A partir dos dados coletados e analisados no presente estudo, conclui-se que o processo de gravimetria é primordial para a elaboração de planos e projetos relacionados ao gerenciamento dos resíduos sólidos, e que o resultado dessa análise pode se apresentar em diferentes formas considerando as características do local em que está sendo realizada. Partindo dessa premissa e após a análise dos dados obtidos, pode-se inferir que para ocorrer o adequado gerenciamento dos resíduos a caracterização quali-quantitativa foi primordial, pois a partir dela foi possível verificar quais resíduos são produzidos em maior escala, a fim de buscar medidas preventivas de redução ou não geração destes, ou ainda a sua correta destinação final. É válido ressaltar que para acontecer a separação apropriada dos resíduos sólidos, principalmente a separação entre os resíduos comuns, orgânicos e rejeitos, é imprescindível a realização de ações de educação ambiental a fim de conscientizar e capacitar todos os envolvidos no processo, desde o descarte, coleta e armazenamento, até a destinação final. Nesse sentido, as medidas expostas neste estudo: diminuição do desperdício de alimentos, reutilização do papel, implementação de campanhas visando a substituição do uso de descartáveis pelo uso de utensílios reutilizáveis, a construção de uma composteira (para destinação dos resíduos orgânicos) e a destinação dos demais resíduos à cooperativas de reciclagem, podem ser adotadas no Campus V da UEPA para o melhor gerenciamento dos resíduos produzidos na instituição.

Além disso, faz-se necessário a realização de estudos que acompanhem a produção de resíduos no Campus V da UEPA para que o plano de gerenciamento seja atualizado conforme as mudanças que ocorrem no dinamismo social presente na Universidade. Outros projetos podem ser desenvolvidos com a finalidade de implementar campanhas de fortalecimento na educação ambiental dos estudantes, funcionários e demais frequentadores da instituição, vide que é um ambiente de constante transformação e de formação de cidadãos. A implementação da composteira pode também ser alvo de novos estudos que contribuam para a universidade e para o conhecimento acadêmico, visto que esta apresenta, desde a sua fabricação até o seu produto final, fontes essenciais de conhecimento acerca da destinação sustentável e adequada de resíduos sólidos.

\section{Referências}

ABRELPE. (2020). Panorama dos Resíduos Sólidos no Brasil 2018/2019. Associação Brasileira de Empresas de Limpeza Pública e Resíduos Especiais ABRELPE. https://abrelpe.org.br/panorama-2020/.

Albuquerque, B. P. (2007). As relações entre o homem e a natureza e a crise sócio-ambiental. Escola Politécnica de Saúde Joaquim Venâncio, Fundação Oswaldo Cruz (Fiocruz).

Andrade, P. S. A. D. M. (2017). A Quarta Revolução Industrial e sua relação com a produtividade atual: Uma revisão da literatura. Trabalho de Conclusão de Curso, Universidade de Brasília. https://bdm.unb.br/handle/10483/17633

Andreoli, C. V., Andreoli, F. N., Trindade, T. V., \& Hoppen, C. (2014). Resíduos sólidos: origem, classificação e soluções para destinação final adequada. https://www.agrinho.com.br/site/wp-content/uploads/2014/09/32_Residuos-solidos.pdf.

ABNT. (2004). NBR 10004: Resíduos sólidos - classificação. Associação Brasileira de Normas Técnicas - ABNT.

ABNT. (2004). NBR 10007: Amostragem de resíduos sólidos. Associação Brasileira de Normas Técnicas - ABNT. 
Baptista, V. F. (2010). A relação entre o consumo e a escassez dos recursos naturais: uma abordagem histórica. Saúde \& Ambiente em Revista, 5(1), 8-14.

Brasil. Lei n. 12.305. (2010). Política Nacional de Resíduos Sólidos; altera a Lei no 9.605, de 12 de fevereiro de 1998; e dá outras providências.

Brasil. Lei n. 9.795. (1999). Dispõe sobre a educação ambiental, institui a Política Nacional de Educação Ambiental e dá outras providências.

Cajaiba, R. L., \& da Silva, W. B. (2017). Composição gravimétrica dos resíduos sólidos de escolas públicas da zona urbana e rural do município de Uruará, PA. SaBios-Revista de Saúde e Biologia, 11(2), 01-06.

Carvalho, R. R., Carvalho, A. A., Silva, M. G. C. D., Carvalho, A. A., Oliveira, M. R. A., \& Cavalcante Neto, A. A. (2012). A compostagem como ferramenta de educação ambiental no Instituto Federal do Maranhão Campus Codó. In VII CONNEPI-Congresso Norte Nordeste de Pesquisa e Inovação.

Costa, F. X., de Lucena, A. M. A., Tresena, N. D. L., Guimarães, F. S., Guimarães, M. M. B., da Silva, M. M. P., \& Guerra, H. O. C. (2006). Estudo qualitativo e quantitativo dos resíduos sólidos do Campus I da Universidade Estadual da Paraíba. Revista de Biologia e Ciências da Terra, 6(1), 1-11.

Cotica, K. J. F., \& Carniatto, I. A relação entre legislação, a educação ambiental e os problemas enfrentados na gestão de resíduos sólidos pelas comunidades rurais. International Journal of Environmental Resilience Research and Science, 2(2).

Coutinho, C., Duarte, A., Carneiro, E., Sampaio, NAS, Silva, JW de J., \& Mühlen, CV (2020). Geração de resíduos sólidos e desenvolvimento de sistema de gestão por meio de análise estatística. Pesquisa, Sociedade e Desenvolvimento, 9 (4), e05942787. https://doi.org/10.33448/rsd-v9i4.2787

Gouveia, N. (2012). Resíduos sólidos urbanos: impactos socioambientais e perspectiva de manejo sustentável com inclusão social. Ciência \& saúde coletiva, $17,1503-1510$

Jacobi, P. (2003). Educação ambiental, cidadania e sustentabilidade. Cadernos de pesquisa, 189-206.

Lima, R. J. G (2018). Manejo e Quantificação dos Resíduos Sólidos Gerados no Campus IV - Rio Tinto da Universidade Federal da Paraíba (UFPB). Anais do $10^{\circ}$ Fórum Internacional de Resíduos Sólidos, João Pessoa, PB, Brasil.

Madeira, D. J. S., \& Martinelli, M. A. B. (2014). Diagnóstico do gerenciamento dos resíduos sólidos na Universidade Tecnológica Federal do Paraná Câmpus Medianeira. Trabalho de Conclusão de Curso, Universidade Tecnológica Federal do Paraná, Medianeira, PR, Brasil.

Marconi, M.A.; Lakatos, E.M. (2003). Fundamentos de metodologia científica. Atlas.

Massukado, L. M. (2004). Sistema de apoio à decisão: avaliação de cenários de gestão integrada de resíduos sólidos urbanos domiciliares. Dissertação de mestrado, Universidade Federal de São Carlos, São Carlos, SP, Brasil.

Mesquita, E. G., Fiuza, M. S. S., \& Sartori, H. J. F. (2011). Gerenciamento de resíduos sólidos: estudo de caso em campus universitário. Revista Construindo, 3(1), 37-45.

Monteiro, J. H. P. (2001). Manual de gerenciamento integrado de resíduos sólidos [Manual]. Instituto Brasileiro de Administração Municipal, Rio de Janeiro, RJ.

Mota, J. C., de Almeida, M. M., de Alencar, V. C., \& Curi, W. F. (2009). Características e impactos ambientais causados pelos resíduos sólidos: uma visão conceitual. Águas Subterrâneas, 1.

Pereira, A. O. K., \& Horn, L. F. D. R. (2009). Relações de consumo: meio ambiente.

Pereira A. S. et al. (2018). Metodologia da pesquisa científica. UFSM. https://repositorio.ufsm.br/bitstream/handle/1/15824/Lic_Computacao_MetodologiaPesquisa-Cientifica.pdf?sequence $=1$

Rodrigues, K., da Silva Mattos, C., Antunes, S. D. S. V., de Souza, I. S., \& Gonçalves, M. S. (2019). Composteira da UTFPR-Campus Campo Mourão: aproveitamento de resíduos e educação ambiental. Anais do $2^{\circ}$ Congresso Sul-Americano de Resíduos Sólidos e Sustentabilidade, Foz do Iguaçu, PR, Brasil.

Rodrigues, S. C. M., Dias, L. A. L., Carvalho, A. C., Fenzl, N., \& do Canto Lopes, L. O. (2019). Os recursos naturais no processo de desenvolvimento econômico capitalista. Semioses, 13(4), 50-68.

Silva, A. D. V., Mendonça, A. W., Marcomin, F. E., Mazzuco, K. T. M., \& Becker, R. R. (2011). Percepção ambiental como ferramenta para processos de educação ambiental na universidade. REMEA-Revista Eletrônica do Mestrado em Educação Ambiental, 27.

Stamm, K., Naszeniak, T., Garlet, T. B., Graichen, D. A., Tremea, E., \& da Silva, M. N. (2019). Diagnóstico, proposta e ações para implementação de composteira no restaurante universitário da Universidade Federal de Santa Maria, Campus Palmeira das Missões. Anais do III Simpósio em saúde e alimentação da Universidade Federal da Fronteira Sul - Campus Chapecó, Chapecó, SC, Brasil.

Verdi, M., \& Pereira, G. R. (2006). A Educação Ambiental na formação de educadores - o caso da Universidade Regional de Blumenau-FURB. REMEA-Revista Eletrônica do Mestrado em Educação Ambiental, 17. 